\title{
Research on Distributed Power Quality Disturbance Detection Based on ILMD
}

\author{
Xiaohui $\mathrm{LIAO}^{1}$, Jingbo XIAO ${ }^{*}$, Zhigang $\mathrm{WANG}^{1}$, Hao WANG ${ }^{1}$, and Kang $\mathrm{NING}^{2}$ \\ ${ }^{1}$ School of Electrical Engineering, Zhengzhou University, Zhengzhou 450001, China \\ ${ }^{2}$ Zhengzhou Airport Xing gang Investment Group Co., Ltd., Zhengzhou 451161, China
}

\begin{abstract}
The local mean decomposition method is effective in analyzing non-linear and non-stationary data, and it is suitable for the detection of power quality disturbance signals. The endpoint effect caused by the method is studied, and the original method is improved for the problem that the disturbance signal cannot be accurately located. An improved Local Mean Decomposition (ILMD) method is proposed. ILMD uses cubic spline interpolation instead of smoothing to obtain local mean function and envelope estimation function. Radial Basis Function (RBF) neural network is used to extend the information at both ends of the data, which improves the endpoint effect. Combined with Hilbert transform, the instantaneous frequency of power quality disturbance signal can be more accurately calculated. The improved method is also applicable to disturbance signals with weak periodic law, and has less requirement for disturbance signal conditions and universal applicability. The effectiveness of ILMD is validated by simulation examples and the measured data of voltage signal at low voltage side of $35 \mathrm{kV}$ bus transformer in a wind farm.
\end{abstract}

\section{INSTRUCTION}

With the technological innovation, the development of distributed generation is in full swing. However, the power quality problems caused by distributed generation cannot be ignored. Due to the need for fast and accurate detection and analysis of disturbance signals in power quality control of distributed generation grid-connected, power quality disturbance has been paid more and more attention. Current detection methods mainly include Stransform, wavelet transform, fractal theory and Hilbert Transform (HT).

There are still many problems that need to be solved urgently. For example, the unnecessary information of Smatrix obtained by S-transform will interfere with or even hide the disturbance characteristics, which will lead to bad influence on the accuracy of signal recognition [12]. In addition, if the fitness of the basis function or the decomposition scale is not appropriate in wavelet transform, it cannot ensure that the best results can be obtained, and when the frequency is small, it is easy to make mistakes [3]. Compared with HT, HT is more mature. At first, the complex signal is decomposed into intrinsic mode function (IMF) by empirical mode decomposition, and then the frequency and amplitude of each IMF are calculated by HT. The perturbation information of the position of the sudden change point can be obtained by analyzing the corresponding data. HT has good adaptability and can be applied in many extreme cases. However, its shortcomings lie in end distortion, mode aliasing, false components and other related drawbacks [4].
Because of the limitation of the method, Smith and other scholars have studied and published the Local Mean Decomposition (LMD) [5], which has the same signal decomposition mode as HT. Comparatively speaking, the iteration frequency of product function component (PF) obtained by LMD decreases, and the accuracy of amplitude and frequency increases. However, LMD algorithm has its own shortcomings. Its instantaneous frequency is limited by the extreme value of the function, so it can not accurately locate the disturbance signal. In addition, the local mean and envelope estimation functions obtained by the smoothing moving method can easily affect the accuracy of data information at both ends, resulting in endpoint effect. Therefore, based on the research of LMD, this paper proposes an improved Local Mean Decomposition (ILMD). ILMD uses Radial Basis Function (RBF) to mirror the symmetric extension of data to improve the endpoint effect of the data. The cubic smoothing method is used to replace the local smoothing method to obtain the local mean and envelope estimation function. ILMD uses HT to obtain the instantaneous frequency of the signal. The reasonable validity of ILMD is demonstrated by simulation examples and measured data.

\section{Analysis of Distributed Power Quality Disturbance Signals}

The access of distributed power sources, such as photovoltaic and wind power, not only brings impact and asymmetric loads, but also changes the power flow of distribution network, and produces harsh problems such

\footnotetext{
* Corresponding author:1431203619@qq.com
} 
as harmonics. Temperature, illumination intensity and uniformity have a deep impact on the output power of photovoltaic cells. Large changes in wind conditions will cause fluctuations in the output power of wind turbines. Therefore, grid-connected wind and photovoltaic systems are prone to voltage fluctuations and flickers. In addition, when wind power is connected to the grid, the maximum probability of start-up operation of constant speed induction wind turbines will cause serious voltage sags. Moreover, since the distributed power source is usually at the end node of the distribution network and is close to the load, the reactive power of the output will cause the load node voltage to shift in an unequal manner. With the deepening of the research on power quality of distributed generation, the detection and analysis of disturbance signal as an important part of optimal governance has been paid more attention. In view of the main consequences of distributed generation access, such as voltage deviation and voltage fluctuation, this paper selects three aspects of voltage sag, voltage fluctuation and frequency offset for simulation analysis.

\section{The Basic Theory of LMD}

LMD actually uses the iterative algorithm according to the envelope characteristics of the signal itself, and decomposes the original data's frequency modulation and envelope signal data from the original disturbance signal in the order of frequency decrement, and then the corresponding product of the two. This is the PF component, which is the time-frequency distribution of the original component [6]. Figure.1 illustrates the detailed decomposition process of the original data.

$$
\begin{gathered}
\text { Beginning } \\
\downarrow \\
\mathrm{i}=0 ; u_{\mathrm{i}}(t)=x(t) \\
\downarrow \\
\mathrm{i}=\mathrm{i}+1, \mathrm{n}=1
\end{gathered}
$$

$\begin{gathered}\text { Finding out the local mean point } \mathrm{n}_{\mathrm{i}} \text { of } u_{\mathrm{i}}(t) \text { and calculating } \\ \text { the average value of any two extreme points, } m_{\mathrm{i}}=\left(n_{\mathrm{i}}+n_{\mathrm{i}+1}\right) / 2\end{gathered}$
$\begin{gathered}\text { Smoothing the adjacent extreme point } \mathrm{m}_{\mathrm{i}} \text { to } \\ \text { obtain the local mean function } \mathrm{m}_{11}(t)\end{gathered}$
Calculating envelope estimates, $\mathrm{a}_{\mathrm{i}}=\left|n_{\mathrm{i}}-n_{\mathrm{i}+1}\right| / 2$
Enveloping estimation function $\mathrm{a}_{11}(t)$ for smoothing adjacent $\mathrm{a}_{\mathrm{i}}$

Fig.1. Flow chart of the LMD decomposition

Repeating the process can obtain the envelope estimation function $a_{1 \mathrm{n}}(t)$, where the premise of the iterative cutoff is:

$$
\lim _{\mathrm{n} \rightarrow \infty} a_{1 \mathrm{n}}(t)=1
$$

The envelope signal $a_{1}(t)$ is obtained by multiplying the envelope estimation function obtained in the iterative process.

$$
a_{1}(t)=a_{11}(t) \cdot a_{12}(t) \cdots a_{1 n}(t)
$$

The product of the frequency modulated signal $S_{1 \mathrm{n}}(t)$ and the envelope signal $a_{1}(t)$ is obtained to calculate the first $\mathrm{PF}$ component of the original signal $u_{i}(t)$, which is set to $\mathrm{PF}_{1}(t)$.

$$
P \mathrm{~F}_{1}(t)=a_{1 t}(t) \mathrm{s}_{1 n}(t)
$$

The $\mathrm{PF}_{1}(t)$ is separated from the original signal to obtain the signal $h_{1}(t)$.

$$
h_{1}(t)=u_{\mathrm{i}}(t)-P \mathrm{~F}_{1}(t)
$$

The above steps are repeated $\mathrm{k}$ times, and the cutoff condition is that $h_{\mathrm{k}}(t)$ is a monotonic function. Furthermore, the original signal consists of two parts, $h_{\mathrm{k}}(t)$ and PF.

$$
u_{\mathrm{i}}(t)=\sum_{i=1}^{k} P \mathrm{~F}_{\mathrm{i}}(t)+h_{k}(t)
$$

\section{Power quality disturbance detection based on ILMD}

\subsection{Endpoint effect analysis and processing}

At the beginning of LMD design, local mean and envelope estimation functions and corresponding curves were obtained by smoothing moving method, and local extreme point information of data was required to be obtained accurately. The problem encountered in the actual operation is that the two endpoints of the data information are not local extreme points, so it is impossible to identify and obtain the necessary characteristic parameters for the algorithm. If neglected, the data deviation will be caused, and the iteration of the algorithm will disturb all the data information [7]. Therefore, to improve the endpoint effect, the accuracy of endpoint extreme value information must be guaranteed.

ILMD uses cubic spline interpolation instead of smooth movement to fit, and RBF neural network method is used to predict the first and last two places of data, obtain the extreme value at the end of data, and then carry out mirror symmetric continuation for data. By processing a section of data and comparing them before improvement, as shown in Figure.2, the results show that the phenomenon of the end-point flying wing at the end of the first and second components of the improved data information can be alleviated, the signal characteristics tend to be stable, and the range of change of the first PF component also converges between -1 and 1.5 , and the end-point effect has been greatly improved. In addition, the protrusion of the third PF component end in the figure is significantly less than that before RBF prediction, which indicates that ILMD does improve the endpoint effect better. 


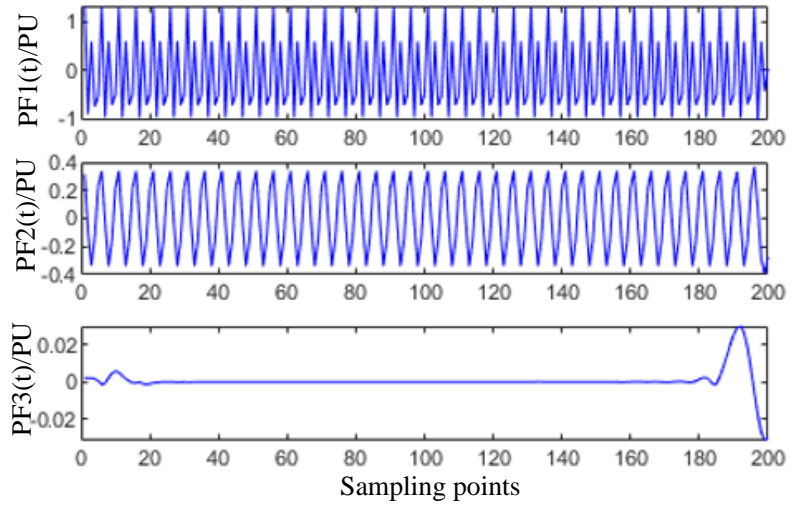

(1) Unimproved Mirror Extension Image
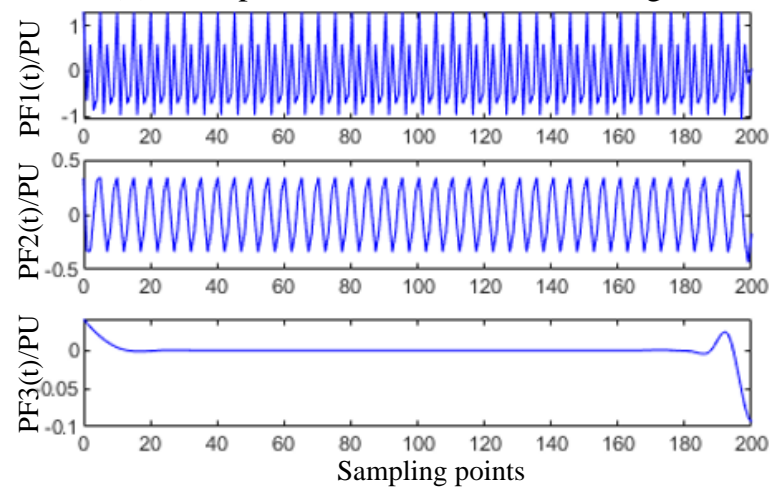

(2) Improved data information image

Fig.2. Comparison of improvement effect

\subsection{Improvement method and process}

ILMD abandons the smooth moving method in LMD, and uses the Mirror symmetric extension method based on RBF to improve the endpoint effect. The cubic spline interpolation method is used to obtain the local mean function and envelope estimation function, and the Hilbert transform is used to obtain the corresponding amplitude-frequency characteristics. The specific flow chart of the method is as follows:

1) Based on the established original data section, the RBF method is used to predict the data segment needed by the two ends of the segment.

2) The extreme point information at both ends of the original function is obtained from the predicted data.

3) Based on the data of the first RBF prediction, the mirror symmetric continuation method is adopted to continue processing outward. The "mirror" is embedded in the position of extreme points at both ends of the data, and the information of extreme points at both ends of the virtual image with the smallest distance in the "mirror" is acquired at the same time.

4) Cubic spline interpolation is used to decompose the predicted and extended data. Here the condition of termination of iteration is changed from "1" to $1-\Delta \leqq$ $\mathrm{a}_{1 \mathrm{n}}(\mathrm{t}) \leqq 1+\Delta$, where $\Delta=0.0001$, which can reduce the number of iterations.

5) In the data after decomposition operation, the data fragments predicted by RBF and extended by mirror symmetry are discarded, and only the part consistent with the length of the original data fragments is retained. The decomposition data obtained in this part of the operation is the data needed for analysis.

6 ) Finally, the corresponding instantaneous frequency information of the above PF components is obtained and analyzed by Hilbert transform.

This method solves the problem of poor positioning accuracy inherent in the original LMD because the instantaneous frequency of disturbance signal is limited by the extreme value. At the same time, ILMD also plays an excellent mitigation role in the end effect. The experimental results show that this method has a good effect.

\section{Example simulation}

\subsection{Voltage sag}

The mathematical model of voltage sag is as follows:

$$
u(t)=U_{m}\left(1+a \times\left(\varepsilon\left(t-t_{1}\right)-\varepsilon\left(t-t_{2}\right)\right)\right) \sin (2 \pi f t)
$$

The value of the coefficient a is $[0.1,0.9]$, and in the sag model should be 0.55 , the value of $t_{1}$ is $0.11 \mathrm{~s}$, the value of $t_{2}$ is $0.04 \mathrm{~s}$, and the noise ratio is 0.04 . Wavelet denoising is used to process the voltage signal, and then the ILMD method is used to analyze the voltage sag signal. The decomposition diagram is shown in Figure.3. Figure. 4 shows the instantaneous frequency and amplitude.
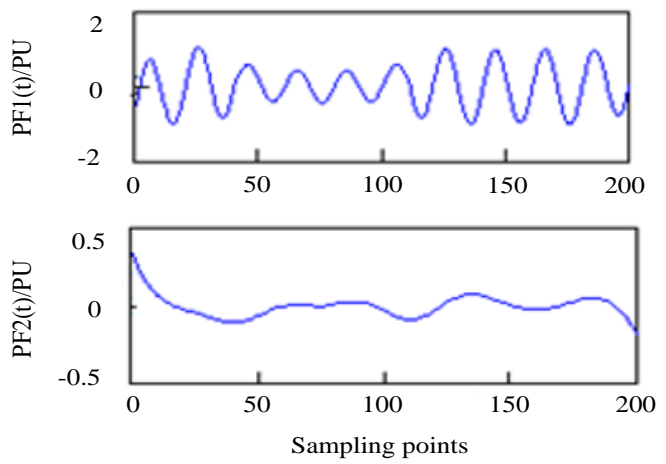

Fig.3. Decomposition image of voltage sag

It can be seen from the results in Fig.4 that the instantaneous frequency of the first PF component does not change significantly, and the start and end times of the PF1 transient amplitude sag are 0.039s and 0.109s. From this, it can be concluded that the analysis and detection results of the voltage dip signal by ILMD are practical and feasible. 

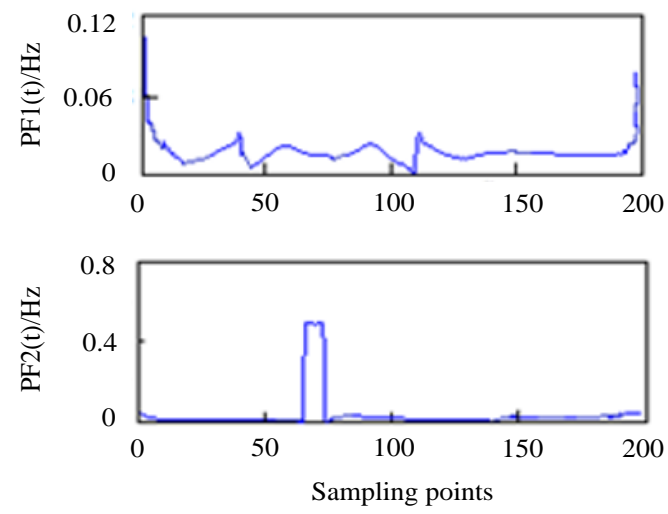

(1) Instantaneous frequency of voltage dip

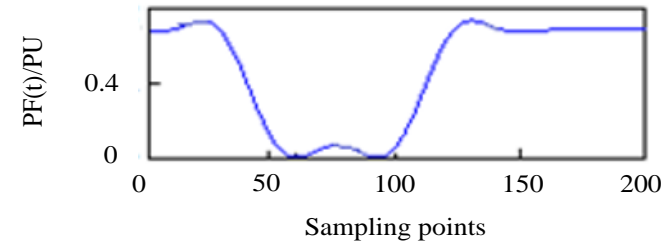

(2) Instantaneous amplitude of voltage dips

Fig.4. Decomposition information of voltage sag

\subsection{Voltage flicker}

The mathematical model of voltage flicker is as follows:

$$
u(t)=U_{m} \sin (2 \pi f t)\left[1 \pm \frac{1}{2} \times \frac{\Delta u}{u} \sin (2 \pi \times \Delta f)\right]
$$

Where, $f$ is $50 \mathrm{~Hz}, \Delta u$ represents voltage fluctuation, and $\Delta f$ represents frequency fluctuation, which is $8.8 \mathrm{~Hz}$. The voltage variation amplitude ratio $\Delta u / u$ is 0.4 . The voltage flicker signal is processed by wavelet noise reduction, and then the flicker signal is analyzed by the above ILMD method, so that the decomposition result can be obtained as shown in Fig.5. As shown in Figure.6, the instantaneous frequency and amplitude are obtained.
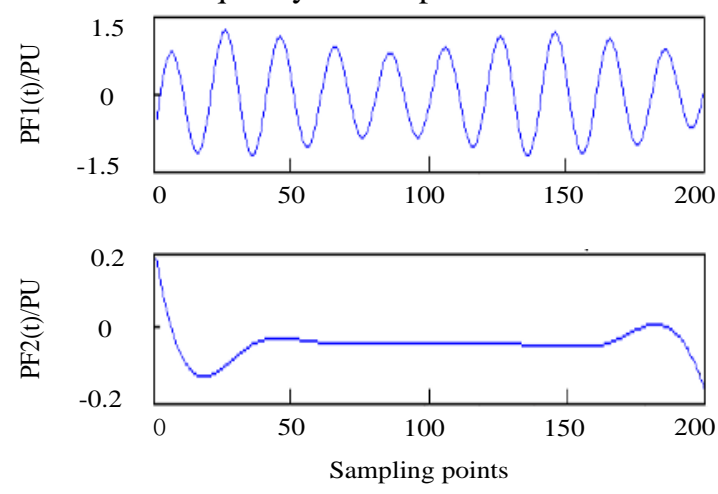

Fig.5. Decomposition image of voltage flicker

It can be seen from the decomposition result of Fig.6 that the instantaneous frequency of the PF1 component of the voltage flicker signal eventually tends to be 0.051 $\mathrm{Hz}$, and there is no periodic fluctuation, and the fluctuation range of the instantaneous amplitude of the flicker signal is $[0.8,1.2]$. Therefore, the results show that the decomposition of voltage flicker by the ILMD method is satisfactory and practical.
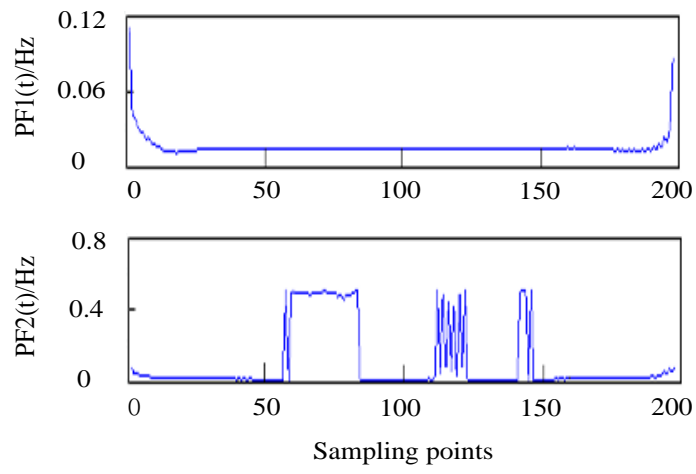

(1) Voltage flicker instantaneous frequency
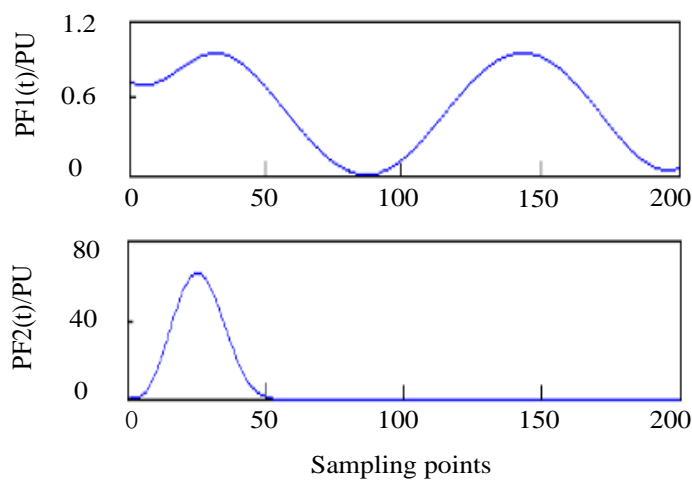

(2) Voltage flicker instantaneous amplitude

Fig.6. Decomposition information of voltage flicker

\subsection{Frequency offset}

The mathematical model of Frequency offset is as follows:

$$
u(t)=a \times\left(\varepsilon\left(t-t_{1}\right)-\varepsilon\left(t-t_{2}\right)\right) \times \sin (2 \pi \Delta f t)
$$

In the formula, the value of $a$ is set to 0.3 , and $\Delta f$ represents the offset of the frequency, and its value is set to $5 \mathrm{~Hz}$, wherein the time parameter $t_{1}$ is $0.12 \mathrm{~s}, t_{2}$ is 0.03 $\mathrm{s}$, and the frequency offset decomposition is as shown in Fig.7.

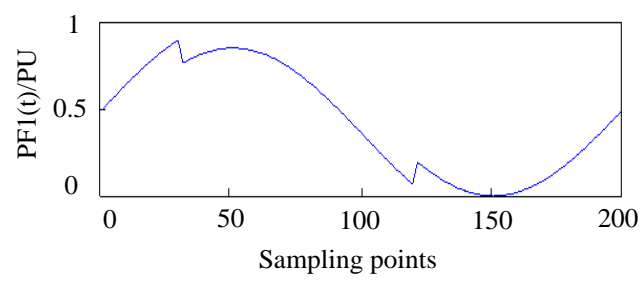

Fig.7. Decomposition image of frequency offset

Fig.8 is the instantaneous frequency and amplitude of the frequency offset signal as determined by the ILMD method.

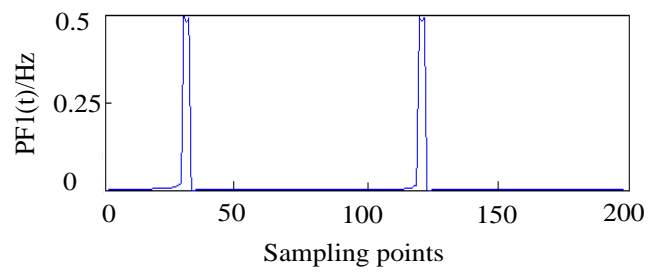

(1) Instantaneous frequency of frequency offset 


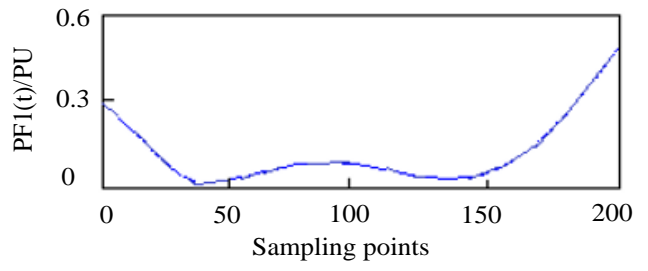

(2) Instantaneous amplitude of frequency offset

Fig.8. Decomposition information of frequency offset

The decomposition information in Fig.8 indicates that the instantaneous time at which the instantaneous frequency of the frequency offset signal is suddenly changed is $0.030 \mathrm{~s}, 0.119 \mathrm{~s}$, and the instantaneous amplitude of the PF1 component periodically changes within the interval $[0.12,0.50]$. Therefore, the results show that the decomposition of the voltage-frequency offset signal by the ILMD method is satisfactory and feasible.

Table 1. Results of disturbance detection.

\begin{tabular}{|c|c|c|c|c|}
\hline \multirow{2}{*}{\multicolumn{2}{|c|}{ Disturbance type }} & \multicolumn{3}{|c|}{ Disturbance positioning } \\
\hline & & Theoretical & $\mathbf{L M D}^{[8]}$ & ILMD \\
\hline \multirow{2}{*}{$\begin{array}{l}\text { Voltage } \\
\text { sag }\end{array}$} & $\begin{array}{l}\text { Starting } \\
\text { time/s }\end{array}$ & 0.040 & 0.038 & 0.039 \\
\hline & $\begin{array}{c}\text { Termination } \\
\text { time/s }\end{array}$ & 0.110 & 0.112 & 0.111 \\
\hline \multirow{2}{*}{$\begin{array}{l}\text { Voltage } \\
\text { flicker }\end{array}$} & $\begin{array}{c}\text { Starting time } \\
/ \mathrm{s}\end{array}$ & 0.850 & 0.846 & 0.848 \\
\hline & $\begin{array}{c}\text { Termination } \\
\text { time/s }\end{array}$ & 1.200 & 1.194 & 1.196 \\
\hline \multirow{2}{*}{$\begin{array}{c}\text { Frequenc } \\
\text { y offset }\end{array}$} & $\begin{array}{c}\text { Starting time } \\
/ \mathrm{s}\end{array}$ & 0.029 & 0.031 & 0.030 \\
\hline & $\begin{array}{c}\text { Termination } \\
\text { time/s }\end{array}$ & 0.120 & 0.118 & 0.119 \\
\hline
\end{tabular}

Table 1 shows the results of power quality disturbance signal detection. As can be seen from Table 1 , this method has better positioning ability and better accuracy than the original LMD. This method can also be used to detect and analyze other power quality disturbance signals.

\section{Analysis of measured data}

This paper takes a disturbance data parameter of low voltage side of $35 \mathrm{kV}$ bus transformer of a wind power plant in Henan as an example to analyze. The sampling frequency is $1000 \mathrm{~Hz}$. The distortion of phase A voltage signal is relatively bad. Fig.9 is the voltage data of this section. The actual measured voltage data of this section is detected and decomposed by ILMD method. From the spectrum analysis, as shown in Fig.10, this part of the voltage data mainly contains. There are three or four harmonics, and the decomposition results are shown in Fig.11.

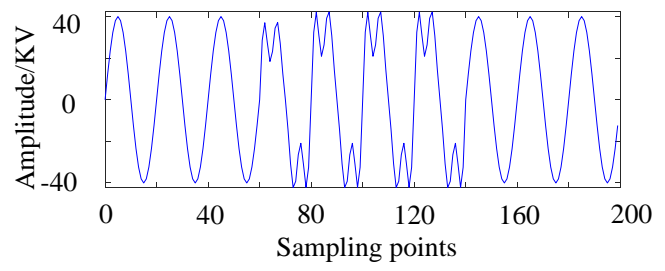

Fig.9. Voltage data of phase A

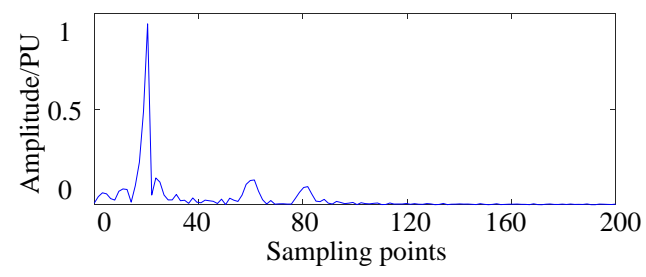

Fig.10. Spectrum analysis of phase A voltage
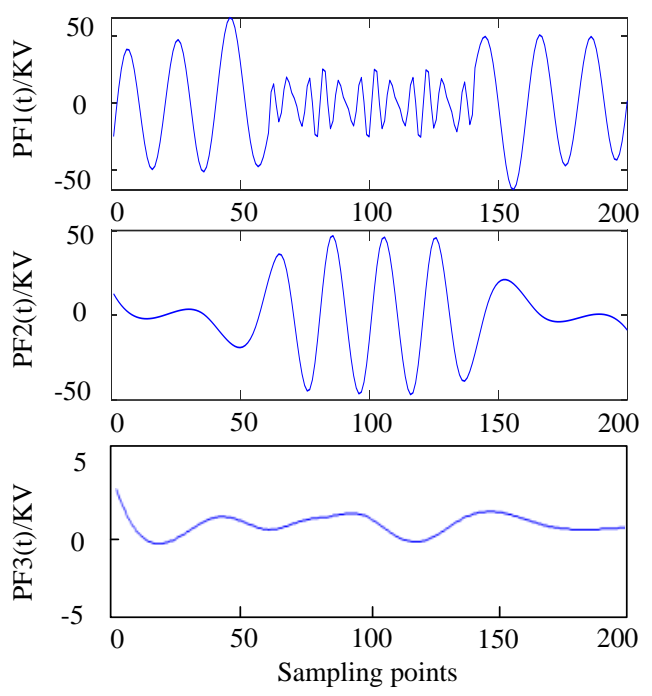

Fig.11. Decomposition diagram of phase A voltage

Figure.12 shows that the instantaneous frequency of the PF1 component of phase A voltage decomposition is higher harmonic, and the results are in good agreement with the spectrum analysis. From Figure.12, it can be concluded that the total time of voltage sag in this part of data is $0.061 \mathrm{~s}$ and $0.142 \mathrm{~s}$. The instantaneous amplitude of PF1 in Figure.13 shows that the high frequency information fluctuates obviously at $0.061 \mathrm{~s}$ and $0.142 \mathrm{~s}$. The image of PF2 shows that the amplitude of low frequency voltage increases temporarily when disturbance occurs at $0.134 \mathrm{~s}$ and $0.142 \mathrm{~s}$ at all times. In addition, the range of low frequency voltage amplitude can be seen from PF3 image as $[0.5,2]$. The results of all the analysis images show that the power quality disturbance analysis method of ILMD is practical, and the results of analysis and detection are accurate and feasible. 

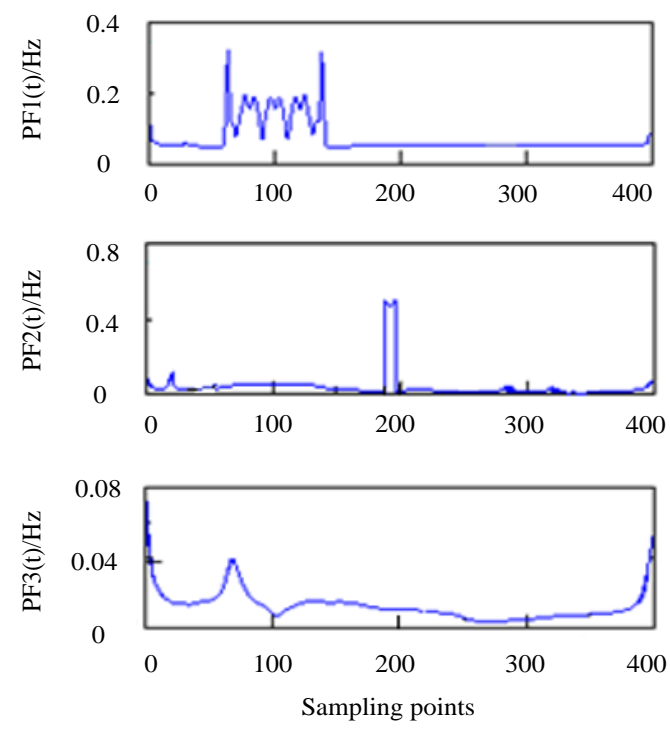

Fig.12. Transient frequency of phase A voltage
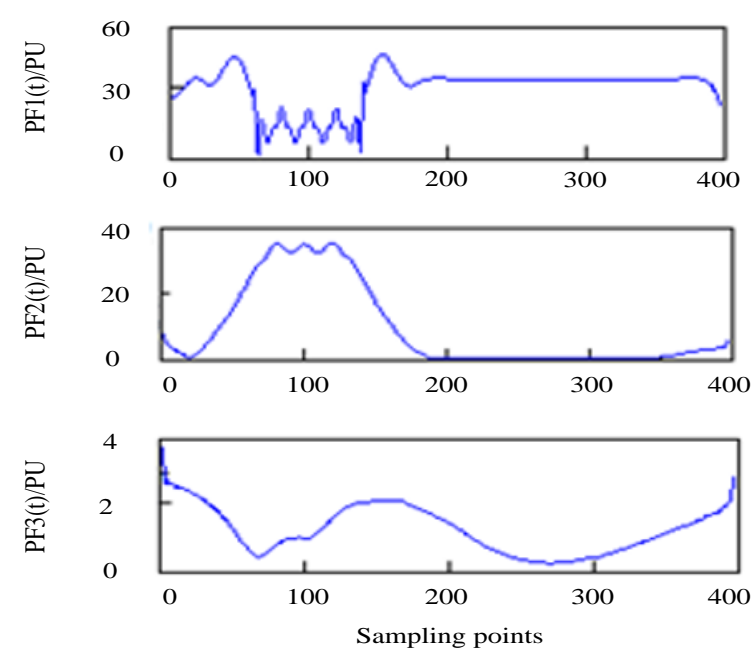

Fig.13. Instantaneous amplitude of phase A voltage

\section{Conclusion}

In this paper, the advantages and disadvantages of various power quality disturbance detection methods are analyzed, and LMD method is improved partially. In particular, the cubic spline difference method is used to replace the smooth moving method in the process of local mean fitting, and the cubic spline difference method is used to calculate the local mean and envelope estimation function. On this basis, the RBF mirror symmetric continuation method is used to improve the endpoint effect, combined with HT. After the instantaneous frequency of power quality disturbance signal is calculated, the shortcoming of inadequate positioning ability of the original method is then avoided. The practical feasibility of ILMD method is verified by using MATLAB simulation examples and measured data analysis, which proves that the accuracy of ILMD method in this paper is higher than that of the original method.

\section{References}

1. Q. Tang, Y. N. Wang, S. Y. Guo, et al, Chin. J. Sci. Instrum 30, pp:166S-1673(2009)

2. M. Biswal, P.K. Dash.IEEE Trans. Circuits Syst 23, 4(2013)

3. D. Camarena-Martinez, M. Valtierra-Rodriguez, C. A. Perez-Ramirez. IRE Trans. Ind. Electron 63, pp:2369-2378(2016)

4. Roberto Moreno, Nancy Visairo, Ciro Núñez. Electr. Power Syst. Res 114 (2014)

5. Smith Jonathan S., J. R. Soc. 2, pp:443-454(2005)

6. R. Kumar, B. Singh, D. T. Shahani, A. Chandra and K. Al-Haddad, IEEE Trans. Ind. Appl 51, pp:12491258 (2015)

7. B. OU Yang, L.Z. YI, J.L. HE. P.S.P.C, 46, pp:143150(2018)

8. K. NING. Power quality analysis of distributed power distribution networks. Master thesis: Zhengzhou university, pp:38-46(2018) 